\title{
Characterization of salt- and surfactant-containing sandy soil extracts by laser light methods
}

\author{
Szilvia Joó ${ }^{1,2}$, Judit Tóth ${ }^{3}$, and Rita Földényi ${ }^{1 *}$ \\ ${ }^{1}$ Department of Earth and Environmental Sciences, University of Pannonia, Egyetem str. 10, H-8200 Veszprém, Hungary \\ ${ }^{2}$ Agricultural Institute, Centre for Agricultural Research, Hungarian Academy of Sciences, Brunszvik str. 2, H-2462 Martonvásár, \\ Hungary \\ ${ }^{3}$ Institute of Materials and Environmental Chemistry, Research Centre for Natural Sciences, Hungarian Academy of Sciences, \\ Magyar tudósok blv. 2, H-1117 Budapest, Hungary
}

Received November 7, 2014; accepted June 15, 2015

A b s t r a c t. The aim of this work was to study how different salt and surfactant solutions influence the particle size distribution and colloidal stability of sandy soil extracts. Particle size distribution was investigated by the laser diffraction method. Extracts were made from the soil - before and after removing its organic content - with solutions of $\mathrm{NaCl}$ or $\mathrm{CaCl}_{2}$ and one cationic and two anionic surfactants. The surfactants influence the particle size distribution of the soil. Due to the use of the $\mathrm{NaCl}$ and surfactant mixtures after removal of organic content, the particle sizes increased compared to the extract of the soil. Colloidal stability was investigated by the laser Doppler electrophoresis method resulting in a zeta potential between -5.63 and $-23.7 \mathrm{mV}$, showing that the extracts were rather instable. Static equilibrium experiments with sodium dodecyl sulphate on sandy soil resulted in an L-type of isotherm with three steps, indicating the formation of more surface layers. Comparison of the adsorption isotherm and the measurements of particle size distribution demonstrated that the particle size changes comparably with the formation of the different layers. The zeta potential of the equilibrated solution reached the region of instability and stability when the initial concentration of sodium dodecyl sulphate was near its critical micelle concentration.

$\mathrm{K}$ e y w o r d s: adsorption, laser diffraction, particle size distribution, soil extract, surfactants

\section{INTRODUCTION}

The particle size distribution (also called grain size distribution or texture) is one of the most important characteristics of soils. Currently, laser diffraction (LD) is increasingly used for particle size distribution (PSD) analysis of sediments and soils. The technique can be very precise and offers advantages of speed and cost over many other methods when used to analyse mixtures of sand, silt,

*Corresponding author e-mail: foldenyi@almos.uni-pannon.hu and clay. There is no standard procedure for determination of soil PSD by the LD method (Arriaga et al., 2006); therefore, data are given in volume percentage. Commonly used methods for determination of PSD (pipette, aerometer etc.) are based on Stokes law and results are expressed in mass percentage. LD has the advantage that small samples can be analyzed accurately, which cannot be carried out by the pipette method (Cooper et al., 1984). The LD method is suitable to investigate also soil suspensions and soil extracts prepared by different methods (Ryżak and Bieganowski, 2010; 2011). Laser diffraction equipment can be used for measurement of particle size in a rather wide range $(0.01-3000 \mu \mathrm{m}), i e$ also in the colloid region. The stability of colloidal solutions can be studied by measurement of electrokinetic (zeta) potential. The alteration in zeta potential is well in agreement with the alteration in physical stability. Minimum zeta potential greater than $|60| \mathrm{mV}$ and greater than $|30| \mathrm{mV}$ is required for excellent and good physical stability, respectively (Malvern Instruments, 2007a). Freitas and Müller (1998) have found that a potential around $|25| \mathrm{mV}$ is just below the critical value; however, in principle, it can still be sufficient for a stable system in combination with the sterically stabilizing effect. Stability of solution affects the sedimentation, adsorption, aggregation, and transportation of contaminants in soils. Better stability means further transport.

The soil solution is a colloidal system containing inorganic (clay minerals) and organic (humic substances) constituents. Humic substances (HS) are special and important components of natural organic matter (NOM) present

(C) 2015 Institute of Agrophysics, Polish Academy of Sciences 
in soil, water, and other geological deposits. Since HS molecules consist of an elongated hydrophobic portion with more anionic (carboxylate) groups, these compounds have amphiphilic character and therefore they can be considered as natural surfactants (Tombácz et al., 1988; Wandruszka, 2000). This character makes them able to form micelles; however, the critical micelle concentration (cmc) of humic acid was estimated to be as high as $10 \mathrm{~g} \mathrm{l}^{-1}$ (Tschapek and Wasowski, 1984). Humic substances are present in natural waters at lower concentration levels (5-100 $\mathrm{mg} \mathrm{l}^{-1}$ ) when aggregates can be formed. These are referred to as pseudomicelles having a hydrophobic interior and a more hydrophilic surface. The hydrophobic moiety in the centre of these aggregates leads to enhanced dissolution ('solubilization') of non-polar contaminants like PAH (Földényi et al., 2013; Senesi, 1993; Wandruszka, 2000). This property of HS can be applied under controlled conditions in remediation of contaminated sites (Nègre et al., 2008) while under natural conditions the same process can lead to mobilization of pollutants (Földényi et al., 2013).

Adsorption is the most important process that plays a role in the retention of pollutants in the bulk phase of soil. The composition and properties of the soil solution $(\mathrm{pH}$ and the nature of electrolytes) are important factors influencing the sorption of different ions and molecules by soil components (Barrow, 1993; Ertli et al., 2004). The adsorption of ions affects the charge and electrokinetic potential of colloidal particles, hence their dispersion-flocculation behaviour. This can have important consequences on soil structural stability, colloid mobility in soils, and groundwater aquifers, as well as suspended sediment features in surface waters (Kretzschmar et al., 1993; McCarthy and Zachara, 1989).

Since processes of pollutant adsorption play a special role in the field of environmental research, there is an increasing demand for investigations of the effect of different chemicals in soil suspensions (Kovács et al., 2004) and extracts. Adsorption processes have determinative importance for chemicals entering the environment. Materials dissolved or suspended in groundwater can self-influence the PSD of soil and the transport of chemicals (Haque and Freed, 1975; Wilson, 1991).

The role of surface-active agents produced by the chemical industry is to dissolve non- or barely watersoluble materials as well as to form stable emulsion or extract; consequently, this type of compounds is likely to be very harmful to the environment. Surfactants in soil may promote the dissolution of different materials; therefore, the transport of contaminants can be faster. This is the case of formation of agents that keep pesticides in the liquid phase and lead to solubilization of barely watersoluble compounds (Földényi et al., 2013; Senesi, 1993). Surfactants can adsorb on the soil surface in more layers (Czinkota et al., 2002); however, there is a contrary process called peptization (Buscall and Ottewill, 1985), wherein these compounds disperse the organic matter content of soil and afterward create a stable colloidal solution (Czinkota et al., 2002).

The soil texture and thus the shape of the PSD curve is a very important sign of other soil properties like hydraulic conductivity, adsorption capacity, etc. If the curve is gently sloping, the hydraulic conductivity of the investigated formation is lower; however, it is more compactable. If the curve is steep, the soil contains a considerable amount of same-sized particles. This means higher hydraulic conductivity and less compactness. Smaller particles can achieve longer distances than larger particles in the soil solution, while they transport adsorbed contaminants. Even the presence of a small fraction of fine particles influences hydraulic conductivity of some porous formations and the possibility of contaminant transport. PSD data can be used for modelling of transport processes (Kovács et al., 2004). Particle size distribution influences the specific surface area of materials and hence the adsorption capacity of the adsorbent (Bänninger et al., 2006).

Our aim was to study how different salt and surfactant solutions influence the particle size distribution and colloidal stability of soil extracts (obtained after centrifugation and filtration). PSD is usually studied in the absence of soil organic matter, but in this case, natural conditions cannot be modelled. Sandy soil was chosen for our investigations because it contains relatively high amounts of soluble humic substances (mostly fulvic acids) at any $\mathrm{pH}$ values.

The influence of the composition of any solution on the PSD of soil extracts has not been studied by other researchers yet; however, it can be very important due to the alteration of various soil properties. Our investigations focused on the role of anionic and cationic surfactants (solutes) in the soil extracts. Three different surface-active materials were selected:

- sodium dodecyl sulphate (SDS), which is used most frequently as a detergent in cosmetics;

- sodium diisopropyl naphthalenesulfonate (Supragil WP), a forming agent in pesticide formulations in agriculture; - and cetyl trimethyl ammonium bromide (CTAB, also known as Cetrimide), an industrial forming agent.

Sodium dodecyl sulphate, which can be used also for soil remediation (Khalladi et al., 2009), and Supragil WP represent the anionic, while CTAB is the cationic type of surfactants. The structure of surfactants is shown in Fig. 1.

PSDs of the extracts were investigated by the laser diffraction method, which is a special application for the investigation of soil texture and is applicable in a wide range of particle size. Colloidal stability of the extracts was studied by the laser Doppler electrophoresis method measuring the zeta potential.

Since humic substances exhibit similar behaviour to surfactants (Tombácz et al., 1988; Wandruszka, 2000), the investigated compounds and HS can form mixed micelles resulting in alteration of the PSD curve and in the stability 


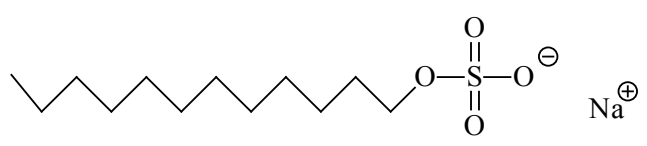

SDS<smiles>CC(C)c1cc2ccccc2c(S(=O)(=O)[O-])c1C(C)C</smiles>

Supragil WP

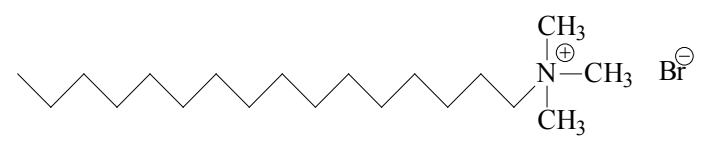

CTAB

Fig. 1. Structure of the surfactants used.

of the colloidal system. Therefore, we intended to make a detailed comparison between the adsorption isotherm generated on the soil and the PSD of the equilibrated soil extracts. For this aim, the surfactant having the most significant effect on changing the particle size distribution was chosen.

\section{MATERIALS AND METHODS}

The sandy soil sample was obtained from the upper $30 \mathrm{~cm}$ layer of the soil in Dabrony, Hungary. Air-dried samples were prepared by grinding in a ball mill for one hour and then sieving under $0.5 \mathrm{~mm}$ particle size. Relevant properties of the studied sandy soil are as follows: specific surface area: $3.85 \mathrm{~m}^{2} \mathrm{~g}^{-1} ; \mathrm{pH}$ : 5.88; TOC (total organic carbon content): $16 \mathrm{mg} \mathrm{C} \mathrm{g}^{-1}$ soil; main minerals: $9.9 \%$ albite, $3.6 \%$ chlorite, $4.8 \%$ mica, $3.8 \%$ microline, $55.3 \%$ quartz, and $18.2 \%$ smectite.

Supragil WP originated from RHODIA Geronazzo (Milan, Italy), while CTAB and SDS were obtained from Reanal Chemical Co. (Budapest, Hungary).

Hydrogen peroxide, $\mathrm{CaCl}_{2}$, and $\mathrm{NaCl}$ salts were purchased from Reanal Chemical Co. Chloroform (for gas chromatography application) was obtained from Spektrum 3D Ltd (Debrecen, Hungary).

For laser diffraction measurements, different sand extracts were prepared using the following treating solutions in distilled water:

0 - distilled water,

$\mathrm{NaCl}-0.1 \mathrm{~mol} \mathrm{l}^{-1}$, $\mathrm{CaCl}_{2}-0.01 \mathrm{moll}^{-1} \mathrm{CaCl}_{2}$, Supragil $-0.1 \mathrm{~g} \mathrm{l}^{-1}$ Supragil WP,

Supragil/ $\mathrm{NaCl}-0.1 \mathrm{~g} \mathrm{l}^{-1}$ Supragil WP and $0.1 \mathrm{~mol} \mathrm{l}^{-1} \mathrm{NaCl}$,
CTAB - $0.1 \mathrm{~g} \mathrm{l}^{-1} \mathrm{CTAB}$,

$\mathrm{CTAB} / \mathrm{NaCl}-0.1 \mathrm{~g} \mathrm{l}^{-1} \mathrm{CTAB}$ in $0.1 \mathrm{~mol} \mathrm{l}^{-1} \mathrm{NaCl}$ solution, SDS $-0.1 \mathrm{~g} \mathrm{l}^{-1} \mathrm{SDS}$, $\mathrm{SDS} / \mathrm{NaCl}-0.1 \mathrm{~g} \mathrm{l}^{-1} \mathrm{SDS}$ in $0.1 \mathrm{~mol} \mathrm{l}^{-1} \mathrm{NaCl}$, $\mathrm{SDS} / \mathrm{CaCl}_{2}-0.1 \mathrm{~g} \mathrm{l}^{-1} \mathrm{SDS}$ in $0.01 \mathrm{~mol} \mathrm{l}^{-1} \mathrm{CaCl}_{2}$.

The soil extracts were prepared as follows:

A extracts: $5 \mathrm{~g}$ soil was shaken with $0.05 \mathrm{l}$ of the abovedescribed solutions for $30 \mathrm{~min}$, centrifuged at $1046 \mathrm{~g}$ for 30 min with a Hettich Universal 32 centrifuge, and allowed to stay overnight at room temperature. The samples were filtered for course particles on filter paper and the extracts were analyzed.

B extracts: Sandy soil was treated with $30 \%$ hydrogen peroxide (until churning of the suspension stopped); then the sample was dried in order to remove the organic content of the soil. The material obtained was suspended in different treating solutions and prepared for LD measurements as described for the A extracts.

The measurements of PSD of the soil extracts were performed by a Mastersizer 2000 (Malvern Instruments) laser diffraction analyser. For measurements, the SM dispersion unit was used: $80-100 \mathrm{ml}$ of distilled water was filled in the unit and stirred at 1200 r.p.m. After background measurements, the sample extract was given into the unit until the obscuration reached $8-10 \%$. For calculation of the PSD, a refractive index of 1.544 (Silica, Quartz) and an adsorption index of 0 were used (Malvern Instruments, 2007b) for sand, and a refractive index of water ie 1.330, was used for the dispersant. The PSDs were characterized by the volume-based frequency curve or by the volume mean diameters $(\mathrm{D})$ of the particles.

The measurements were carried out with three replicates.

The zeta potential of the A and B soil extracts was determined and the extracts made with different concentrations of SDS (0.1-1000 mg l-1 $^{-1}$ solutions for adsorption experiments) were filtered through a $0.45 \mathrm{~mm}$ membrane and measured by a ZetaSizer Nano ZS (Malvern) device. Measurements were carried out at $25^{\circ} \mathrm{C}$ with capillary cuvette and the Smoluchowski equation was used for the calculation (Cieśla et al., 2011). The measurements were carried out with three replicates.

Adsorption of SDS dissolved in the $0.01 \mathrm{~mol} \mathrm{l}^{-1} \mathrm{CaCl}_{2}$ solution was investigated on sandy soil by static equilibrium experiments at $25^{\circ} \mathrm{C}$ in triplicate runs. Exactly known amounts of the soil $(7 \mathrm{~g})$ were weighed and left to stand in $5 \mathrm{ml} 0.01 \mathrm{~mol} \mathrm{l}^{-1} \mathrm{CaCl}_{2}$ for 24 hours. $65 \mathrm{ml}$ of SDS solution (from 10 to $1000 \mathrm{mg} \mathrm{l}^{-1}$ ) was added to the suspensions; the flasks were then shaken $\left(150 \mathrm{~min}^{-1}\right)$ for one hour and left to stand overnight for equilibration. Equilibrium phases were separated by centrifugation (at $1046 \mathrm{~g}$ for $30 \mathrm{~min}$ ) and then by filtration.

The concentration of SDS in the supernatant liquid was determined by two-phase titration described elsewhere (Patzkó, 1996). Cationic surface-active agents can be titrated 
by anionic surfactants and vice versa. The concentration of SDS was analyzed in a shaking graduated cylinder in the presence of the methylene blue indicator. The samples were titrated by a $0.001 \mathrm{~mol} \mathrm{l}^{-1} \mathrm{CTAB}$ solution until methylene blue colour disappeared in the organic (chloroform) phase. The equilibrium concentration was determined by the calibration curve (from 0 to $1000 \mathrm{mg} \mathrm{l}^{-1}$ ) and the specific adsorbed amount ( $q, \mathrm{mg} \mathrm{SDS} \mathrm{g}^{-1}$ soil) was calculated by the following equation:

$$
q=\frac{\left(c_{0}-c\right) V}{m},
$$

where: $V$ is the volume of the equilibrium solution $(0.0701)$, $c_{0}$ is the initial and $c$ is the equilibrium concentration of the investigated compound ( $\left.\mathrm{mg} \mathrm{l}^{-1}\right)$, and $m$ is the weighed amount of the air dry soil (g).

The isotherm was fitted by Eq. (2) as suggested by Czinkota et al. (2002):

$$
q=\sum_{i=1}^{s}\left\{\frac{q_{T_{i}} K_{i}\left(c-b_{i}+\left|c-b_{i}\right|\right)^{n_{i}}}{2^{n_{i}}+K_{i}\left(c-b_{i}+\left|c-b_{i}\right|\right)^{n_{i}}}\right\},
$$

where: $s$ - the number of steps of the isotherm, $q_{T i}-$ adsorption capacity, $K_{i}$ - equilibrium constant, $b_{i}$ - critical concentration limit, $n_{i}$ - average degree of association - all assigned to the $i$ th step of the curve.

The PSD of the equilibrated systems (similar to soil extracts) was also investigated by the laser diffraction method described above. All the measurements were carried out with three replicates.

\section{RESULTS AND DISCUSSION}

The laser diffraction method was used for the measurement of PSD of soil extracts. The PSD of the A extracts without salt showed bimodal distributions (Fig. 2a).

Surfactants (Supragil, CTAB and SDS) influenced the PSD; the ratio of particles between 1 and $200 \mu \mathrm{m}$ decreased in all three cases compared to the simple water

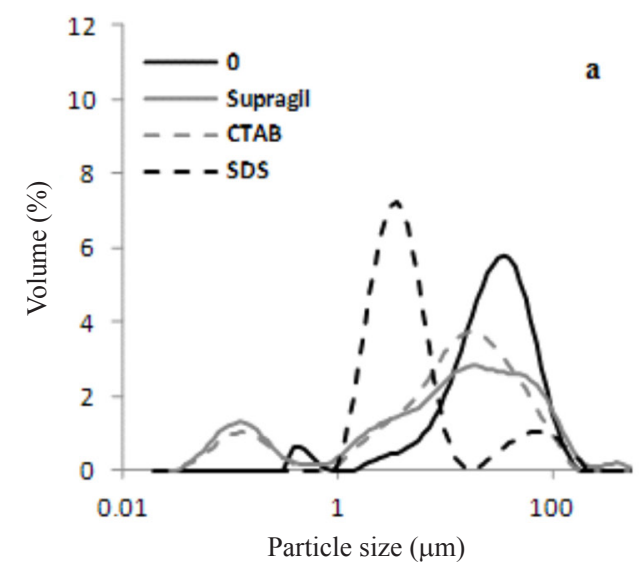

extract (0). Supragil and CTAB showed a similar effect: the gently sloping curve indicated a low percent of very small particles $(\varnothing \leq 1 \mu \mathrm{m})$ but a higher amount of particles occupied the 1-200 $\mu \mathrm{m}$ of the particle size range. The PSD was most significantly shifted to smaller particle sizes in the SDS extract. Addition of surfactants affects the hydraulic conductivity of porous media like soils. The interaction can be very different depending on the surfactant and soil characteristics, the surfactant concentration, and the influence of ionic strength. Allred and Brown (1994) investigated the hydraulic conductivity changes of Daugherty (USA) sand treated with different nonionic, anionic, and cationic type surfactants. They have found that in the case of an anionic surfactant (the concentration of the surfactant was $0.5 \mathrm{~mol} \mathrm{~kg}^{-1}$ ), eg SDS, the hydraulic conductivity decreased significantly, and the SDS caused a high dispersion ratio of the particles. On the other hand, they found that when two different cationic surfactants were applied, the hydraulic conductivity decreased also significantly but the particle mobility was not affected compared to the untreated soil. Mohamed (2014) investigated the interaction between ethanediyl1,2-bis (dimethyldodecylammonium chloride) as a cationic surfactant and sandy soil. It was found that, due to a hydraulic conductivity decrease, the mean weight diameter of the soil increased. In the process of mobilization of small particles from soils, the hydraulic conductivity change is very important, but the PSD cannot be predicted when only this parameter is taken into account.

The added solution electrolytes affect also the hydraulic conductivity and, consequently, the dispersion rate and the average size of the investigated soil extracts. In our case, at the $0.1 \mathrm{~mol} \mathrm{l}^{-1}$ concentration of $\mathrm{NaCl}$ in the surfactant solutions $(\mathrm{NaCl}$, Supragil/ $\mathrm{NaCl}, \mathrm{CTAB} / \mathrm{NaCl}$ and $\mathrm{SDS} /$ $\mathrm{NaCl})$, the PSD and the volume mean diameter changed significantly in two cases $(\mathrm{NaCl}$ and Supragil/ $/ \mathrm{NaCl})$ compared to samples treated without $\mathrm{NaCl}$. The PSD can be seen in Fig. 2b, and the volume mean diameters $\left(\mathrm{D}_{1}\right)$ of the soil $\boldsymbol{A}$ extracts are summarized in Table 1.The PSD of

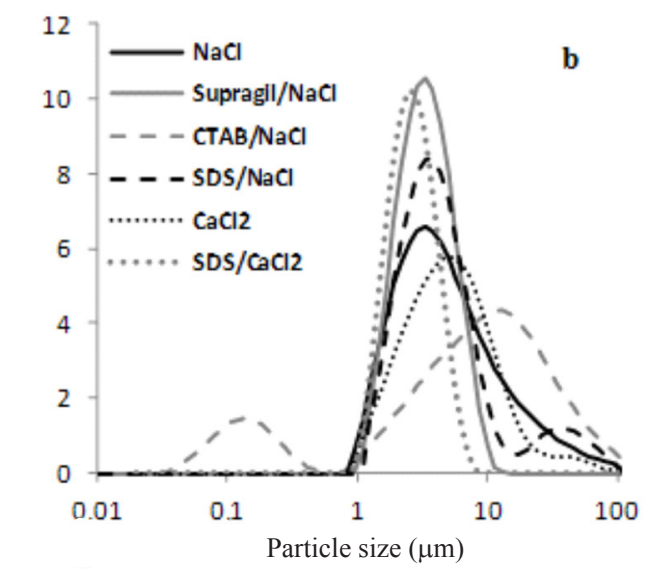

Fig. 2. PSD of sandy soil A extracts treated with a surfactant dissolved in: a - water or in $0.1 \mathrm{~mol} \mathrm{l}^{-1} \mathrm{NaCl}_{1} \mathrm{~b}-0.01 \mathrm{~mol} \mathrm{l^{-1 }} \mathrm{CaCl}_{2}$ solutions. 
T a b l e 1. Volume mean diameters of extracts prepared by different solutions before $\left(D_{1}\right)$ and after $\left(D_{2}\right) \mathrm{H}_{2} \mathrm{O}_{2}$ treatment of sandy soil

\begin{tabular}{lcc}
\hline \multirow{2}{*}{ Solution } & \multicolumn{2}{c}{ Extract $(\mu \mathrm{m})$} \\
\cline { 2 - 3 } 0 & $\mathrm{~A}\left(\mathrm{D}_{1}\right)$ & $\mathrm{B}\left(\mathrm{D}_{2}\right)$ \\
\hline $\mathrm{NaCl}$ & 37.44 & 0.61 \\
$\mathrm{CaCl}_{2}$ & 8.90 & 3.89 \\
Supragil & 14.49 & 63.85 \\
Supragil/NaCl & 31.60 & 3.84 \\
$\mathrm{CTAB}$ & 3.94 & 38.14 \\
$\mathrm{CTAB} / \mathrm{NaCl}$ & 23.10 & 10.29 \\
$\mathrm{SDS}$ & 15.87 & 27.61 \\
$\mathrm{SDS} / \mathrm{NaCl}$ & 14.43 & 11.98 \\
$\mathrm{SDS} / \mathrm{CaCl}$ & 9.14 & 68.10 \\
\hline
\end{tabular}

$\mathrm{CTAB}$ and $\mathrm{CTAB} / \mathrm{NaCl}$ as well as $\mathrm{SDS}$ and $\mathrm{SDS} / \mathrm{NaCl}$ is almost the same as when the surfactant dissolved only in water was used for the treatment (ie without $\mathrm{NaCl}$ ). In the case of anionic surfactants (Supragil $/ \mathrm{NaCl}$ and $\mathrm{SDS} / \mathrm{NaCl}$ ), the average particle size was under $10 \mu \mathrm{m}$, similar as for salted water $(\mathrm{NaCl})$. At the $0.01 \mathrm{~mol} \mathrm{l}^{-1}$ concentration of $\mathrm{CaCl}_{2}$ for the treatment $\left(\mathrm{CaCl}_{2}\right.$ extract), the PSD obtained is very similar to the $\mathrm{NaCl}$ extract with lower shifting to higher particle sizes. At the application of the electrolyte together with SDS ( $\left.\mathrm{SDS} / \mathrm{CaCl}_{2}\right)$, a similar effect was observed to that for the Supragil/ $\mathrm{NaCl}$ and $\mathrm{SDS} / \mathrm{NaCl}$ extracts ie the average size decreased below $10 \mu \mathrm{m}$.

The organic materials were removed from the sand before the preparation of the $\mathrm{B}$ extracts to investigate the influence of the organic matter on immobilization of soil particles. After removing the organic materials, the

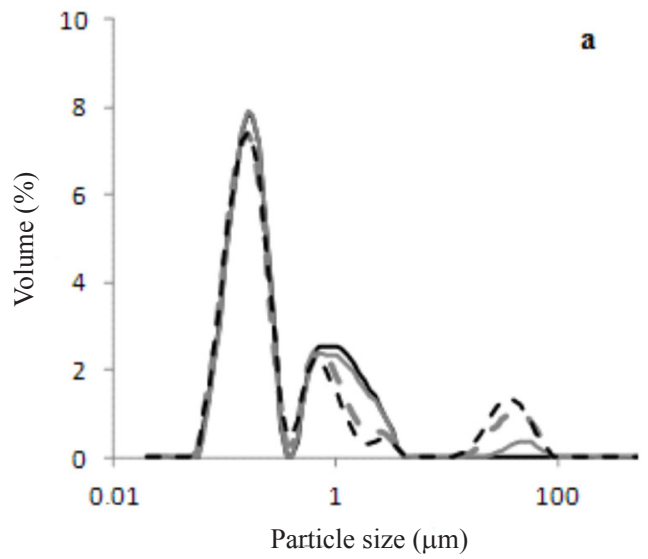

treatment without electrolytes resulted in very similar PSDs of the different extracts ( 0 , Supragil, CTAB, and SDS in Fig. 3a).

When the treatment was carried out in the presence of electrolytes (Fig. 3b), the PSDs changed significantly compared to the $\boldsymbol{A}$ extracts (Fig. 2b). In the case of samples $0, \mathrm{NaCl}$, Supragil, CTAB and SDS, the particle size $\left(\mathrm{D}_{2}\right.$ data in Table 1) was shifted to smaller values after the organic matter was removed. If the treating solutions contained electrolytes and surface-active materials together, an increasing tendency of particle size was observed compared the particle size of the Supragil/ $\mathrm{NaCl}, \mathrm{CTAB} / \mathrm{NaCl}$, SDS/ $\mathrm{NaCl}$, and $\mathrm{SDS} / \mathrm{CaCl}_{2}$ in the A and $\mathrm{B}$ extracts $\left(\mathrm{D}_{1}\right.$ and $\mathrm{D}_{2}$ data in Table 2). Increasing particle size was also found when $\mathrm{CaCl}_{2}$ was used alone for the treatment. The increase in particle size was remarkable when anionic surfactans were used (Supragil/ $\mathrm{NaCl}, \mathrm{SDS} / \mathrm{NaCl}$, and $\mathrm{SDS} / \mathrm{CaCl}_{2}$ ); the values were higher than for the extract with water (0) in the A extract. In the absence of organic matter, the investigated surfactant together with salt promoted the formation of aggregates (flocculation) and not dispersion. The experiments demonstrated that both surfactants and $\mathrm{NaCl}$ used separately promote the dispersion of smaller inorganic particles but they can repeal this effect when used together.

Since zeta potential $(\zeta)$ gives valuable information about the colloidal stability of the system, the A and B soil extracts were studied in this aspect. It was found that these extracts were mostly unstable (Table 2). The smallest stability was obtained between the A extracts when $\mathrm{NaCl}$ was used alone for the preparation of the extract ( $\zeta_{1}$ data in Table 2). Considering the surfactant-containing samples, it can be seen that, in the presence of electrolytes, the instability of the system is very similar to that of the extract made with pure water (a decreasing tendency can be observed in $\zeta_{1}$ data but the standard deviation of the data ranged between 0.99 and 3.80). The rather high ionic strength may cause phase separation in the HS containing system (Tombácz

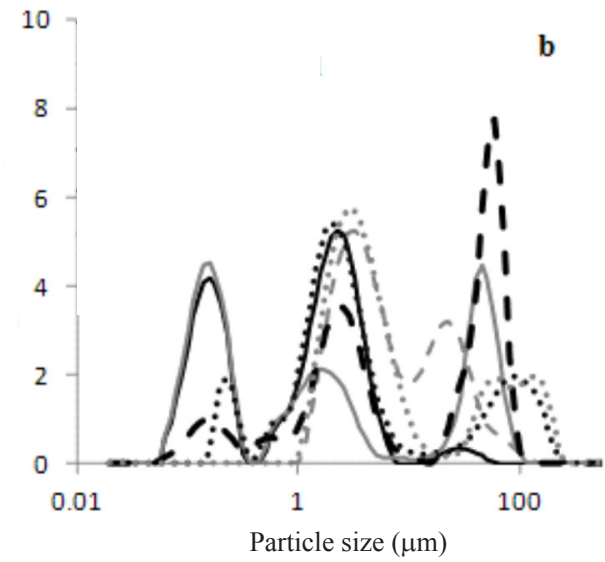

Fig. 3. PSD of sandy soil $\mathrm{B}$ extracts treated with a surfactant dissolved in: a - water or in $0.1 \mathrm{~mol} \mathrm{l}^{-1} \mathrm{NaCl}, \mathrm{b}-0.01 \mathrm{~mol} \mathrm{l}^{-1} \mathrm{CaCl}_{2}$ solutions. Explanations as in Fig. 2. 
T a b l e 2. Zeta potential of A and B sandy soil extracts

\begin{tabular}{|c|c|c|}
\hline \multirow{2}{*}{ Solution } & \multicolumn{2}{|c|}{ Extract $(\mu \mathrm{m})$} \\
\hline & $\mathrm{A}\left(\zeta_{1}\right)^{*}$ & $\mathrm{~B}\left(\xi_{2}\right)^{*}$ \\
\hline 0 & -16.6 & -15.9 \\
\hline $\mathrm{NaCl}$ & -9.37 & -6.2 \\
\hline $\mathrm{CaCl}_{2}$ & -13.2 & -12.3 \\
\hline Supragil & -18.2 & -13.4 \\
\hline Supragil/NaCl & -15.4 & -8.2 \\
\hline CTAB & -12.2 & -11.1 \\
\hline $\mathrm{CTAB} / \mathrm{NaCl}$ & -11.2 & -8.81 \\
\hline SDS & -23.7 & -13.6 \\
\hline $\mathrm{SDS} / \mathrm{NaCl}$ & -16.5 & -5.63 \\
\hline $\mathrm{SDS} / \mathrm{CaCl}_{2}$ & -22.7 & -6.81 \\
\hline
\end{tabular}

*Standard deviation.

et al., 2001) at the surfactant concentration applied. SDS and the SDS/ $\mathrm{CaCl}_{2}$ extracts resulted in the highest stability compared to the other solutions. In all cases of the $\mathrm{B}$ extracts, the zeta potential $\left(\zeta_{2}\right.$ data in Table 2$)$ decreased compared to the A extracts, especially significant changes were observed in the extract containing SDS.

Since the PSD curves of the investigated surfactants indicated that SDS might cause the most significant environmental impact, adsorption studies were carried out with this compound. SDS was dissolved in a $0.01 \mathrm{~mol} \mathrm{l}^{-1}$ $\mathrm{CaCl}_{2}$ solution and the concentration as well as the PSD was determined in the liquid phase after equilibration with sandy soil (see Materials and methods). In order to clarify the relationship between the adsorption of the surfactant and the particle sizes measured in the extracts, the results are shown in the same figure (Fig. 4). In Table 3, the initial (applied) and equilibrium (measured) concentration of SDS are described.

The adsorption curve has three steps and, according to the shape of its initial part, it can be classified as a Langmuir type (L-type) of the isotherm indicating that the solute and the water molecules do not compete with each other to the active sites (Giles et al., 1960). This first step can be explained by hydrophobic interaction between the solute and the hydrocarbon (due to HS) as well as siloxane (due to quartz) chains forming hydrophobic binding sites on the surface (Johnston and Tombácz, 2002; Majzik and Tombácz, 2007). According to the work of Czinkota et al. (2002), further steps of an isotherm must be the result of associates either formed in the solution and adsorbed afterwards or formed by the molecules adsorbed. In this case, the so-called multilayer adsorption occurs. Since SDS being an anionic surfactant and humic substances having also amphiphilic character are present, the adsorption process needs more detailed discussion where the PSD investigations will be taken also into account (Fig. 4, Table 3).

The specific adsorbed amount $(q)$ increased suddenly above a $32 \mathrm{mg} \mathrm{l}^{-1}$ equilibrium concentration (c) when also the particle size became significantly larger. These results were obtained when $100 \mathrm{mg} \mathrm{l}^{-1}\left(c_{0}\right)$ SDS was used for equilibration (Table 3 ). The critical micelle concentration (CMC) of SDS determined in $0.01 \mathrm{~mol} \mathrm{l}^{-1} \mathrm{CaCl}_{2}$ (Sebestyén, 2008) is just equal to this particular initial concentration when the second step of the isotherm is started. In this case, not only single molecules but also micelles of the surfactant can be adsorbed on the soil, resulting in more negative charge repelling the dissociated carboxylic groups of HS on the surface. This repulsing effect leads to solubilization

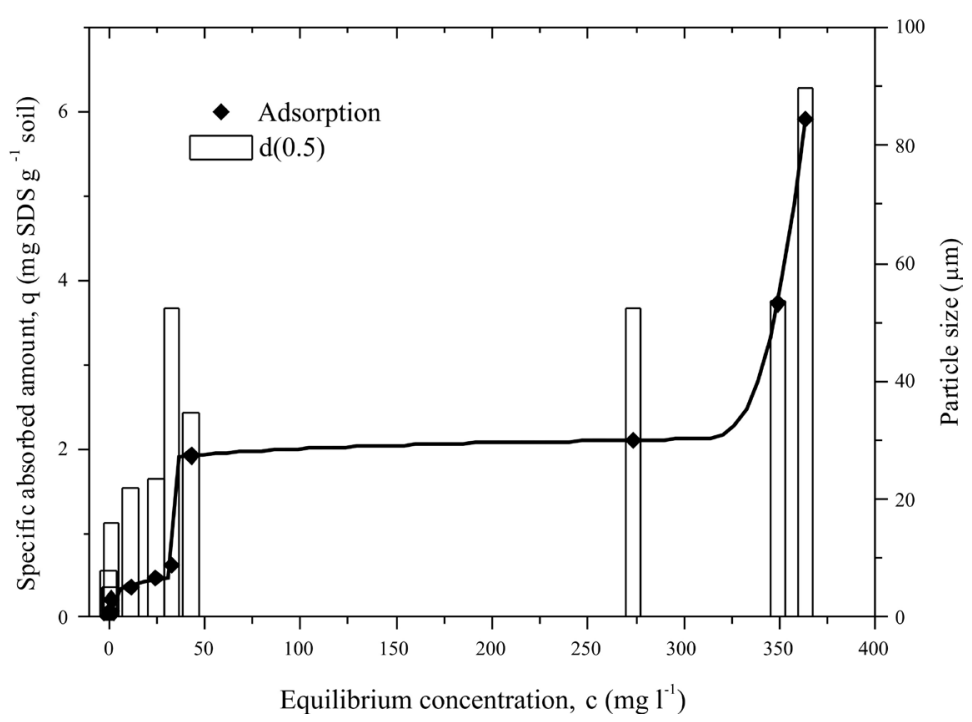

Fig. 4. Adsorption isotherm of SDS on sandy soil and d(0.5) mean particle size determined in the equilibrated liquid phase. 
T a b l e 3. Initial ( $c_{0}$, applied) and equilibrium ( $c$, measured) concentration of SDS during the adsorption experiment

\begin{tabular}{ccccccccccc}
\hline \multicolumn{10}{c}{ SDS concentration $\left(\mathrm{mg} \mathrm{l}^{-1}\right)$} \\
\hline$c_{0}$ & 0 & 10 & 25 & 50 & 75 & 100 & 250 & 500 & 750 & 1000 \\
$c$ & 0 & 0.72 & 1.44 & 11.5 & 24.5 & 32.7 & 42.8 & 274 & 349 & 363 \\
\hline
\end{tabular}

of humic substances that are able to form also micelles; thus, big aggregates can be obtained above the $350 \mathrm{mg} \mathrm{l}^{-1}$ equilibrium concentration of SDS (when $c_{0}$ is above $750 \mathrm{mg}$ $1^{-1}$, Table 3 ), and the third step of the isotherm is evolving.

The $\zeta$ of sandy soil extracts obtained with different initial concentrations of SDS solutions are as follows: $-20.8 \mathrm{mV}$ $\left(0.1 \mathrm{mg} \mathrm{l}^{-1}\right) ;-19.5 \mathrm{mV}\left(1 \mathrm{mg} \mathrm{l}^{-1}\right) ;-23.7 \mathrm{mV}\left(100 \mathrm{mg} \mathrm{l}^{-1}\right)$; and $-46.6 \mathrm{mV}\left(1000 \mathrm{mg} \mathrm{l}^{-1}\right)$. Comparison of these results to the PSD measurements (Fig. 4) as well as to the $\zeta$ values of extracts made by SDS showed that the zeta potential of equilibrated solution reached the limit between instability and stability $(-23.7 \mathrm{mV})$ when the initial concentration was close to CMC (100 $\left.\mathrm{mg} \mathrm{l}^{-1}\right)$, which proved to be of special value considering the newly formed surface.

In our surfactant-containing system, either homogenous micelles (SDS-SDS, HS-HS) or mixed micelles (SDS-HS) can be formed (Tombácz et al., 1988) due to the solubilization process (Földényi et al., 2013; Senesi, 1993), which is caused here by the anionic surfactant. Mixed micelles are the result of interaction between the hydrophobic moieties of the surfactant and that of HS. These associates also contribute to the increased particle size measured in the equilibrated system during adsorption (Fig. 4). Since the homogenous micelles of a surfactant have higher CMC when also mixed associates can be formed (Nylander et al., 2006), it can be pointed out that the third step of the isotherm starts above this higher value ( $c>300 \mathrm{mg} \mathrm{l}^{-1}$, Fig. 4).

\section{CONCLUSIONS}

1. According to the results of particle size distribution measurements of sandy soil extracts, it can be pointed out that the surfactants significantly influenced the particle size distribution.

2. Without electrolytes, peptization of organic material was especially enhanced by sodium dodecyl sulphate, which is used widely as a detergent.

3. The experiments demonstrated that both surfactants and sodium chloride used separately promote the dispersion of smaller inorganic particles but they can repeal this effect when used together.

4. In the absence of organic matter, the investigated surfactant treatment resulted in very similar PSDs when electrolytes were not used.

5. In the absence of organic matter, the investigated surfactant together with the salt promoted the formation of aggregates (flocculation) and not the dispersion.
6. The resulted in adsorption of sodium dodecyl sulphate on sandy soil showed an isotherm curve with three steps and, according to the shape of its initial part. It was classified as a Langmuir type (L-type) of the isotherm.

7. Comparison of the adsorption isotherm and the particle size distribution data for sodium dodecyl sulphate revealed significant changes in the isotherm as well as in the particle size distribution at similar values of initial concentration.

\section{REFERENCES}

Allred B. and Brown O.G., 1994. Surfactant-induced reductions in soil hydraulic conductivity. Ground Water Monit. Rem., 174, 174-184.

Arriaga F.J., Lowery B., and Mays M.D., 2006. A fast method for determining soil particle size distribution using a laser instrument. Soil Sci., 171, 663-674.

Barrow N.J., 1993. Effects of surface heterogeneity on ion adsorption by metal oxides and by soils. Langmuir, 9, 2606-2611.

Bänninger D., Lehmann P., and Flühler H., 2006. Modeling the effect of particle size, shape and orientation of light transfer through porous media. Eur. J. Soil Sci., 57, 906-915.

Buscall R. and Ottewill R.H., 1985. The stability of polymer lattices. In: Polymer Colloids. (Eds R. Buscall, T. Corner, J.F. Stageman). Elsevier Applied Sci. Publishers, London- New York.

Cieśla J., Bieganowski A., Janczarek M., and Urbanik-Sypniewska T., 2011. Determination of the electrokinetic potential of Rhizobium leguminosarum bv trifolii Rt24.2 using Laser Doppler Velocimetry - A methodological study. J. Microbiol. Meth., 85, 199-205.

Cooper L.R., Haverland R.L., Hendricks D.M., and Knisel W.G., 1984. Microtic particle-size analyser: an alternative particle-size determination method for sediment and soils. Soil Sci., 138, 138-146.

Czinkota I., Földényi R., Lengyel Zs., and Marton A., 2002. Adsorption of propisochlor on soils and soil components Equation for multi-step isotherms. Chemosphere, 48, 725-731.

Ertli T., Marton A., and Földényi R., 2004. Effect of $\mathrm{pH}$ and the role of organic matter in the adsorption of isoproturon on soils. Chemosphere, 57, 771-779.

Földényi R., Tóth Z., Samu Gy., and Érsek Cs., 2013. Study of sorption of two sulfonylurea type of herbicides and their additives on soils and soil components. J. Environ. Sci. Heal. B., 48, 758-766.

Freitas C. and Müller R.H., 1998. Effect of light and temperature on zeta potential and physical stability in solid lipid nanoparticle (SLN $\left.{ }^{\mathrm{TM}}\right)$ dispersions. Int. J. Pharm, 168, 221-229. 
Giles C.H., MacEwan T.H., Nakhva S.N., and Smith D., 1960. Studies in adsorption. Part XI. A system of classification of solution adsorption isotherms, and its use in diagnosis of adsorption mechanisms and in measurement of specific surface areas of solids. J. Chem. Soc., 3, 3973-3993.

Haque R. and Freed V.H., 1975. Environmental Dynamics of Pesticides. Plenum Press, New York-London.

Johnston C.T. and Tombácz E., 2002. Surface chemistry of soil minerals. In: Soil Mineralogy with Environmental Applications. (Eds J. Dixon, D. Schulze). SSSA Book Ser. 7, SSSA, Madison, USA.

Khalladi R., Benhabiles O., Bentahar F., and Moulai-Mostefa N., 2009. Surfactant remediation of diesel fuel polluted soil. J. .Hazard. Mater., 164, 1179-1184.

Kovács B., Czinkota I., Tolner L., and Czinkota Gy., 2004. The determination of particle size distribution (PSD) of clayey and silty formations using the hydrostatic method. Acta Mineralogica-Petrographica, 45, 29-34.

Kretzschmar R., Robarge W.P., and Weed S.B., 1993. Flocculation of kaolinitic soil clays: Effects of humic substances and iron oxides. Soil Sci. Soc. Am. J., 57, 1277-1283.

Majzik A. and Tombácz E., 2007. Interaction between humic acid and montmorillonite in the presence of calcium ions I. Interfacial and aqueous phase equilibria: Adsorption and complexation. Org. Geochem., 38, 1319-1329.

Malvern Instruments, 2007a. Sample Dispersion and Refractive Index Guide. MAN0396 1.0, Malvern Instruments Ltd., Malvern, UK.

Malvern Instruments, 2007b. ZetaSizer Nano Series User Manual. Malvern Instruments Ltd., Malvern, UK.

McCarthy J.F. and Zachara J.M., 1989. Subsurface transport of contaminants. Environ. Sci. Technol., 23, 496-502.

Mohamed M.M., 2014. The influence of cationic surface active agent on physical properties of some Egyptian soils. J. Am. Sci., 10, 92-95.

Nègre M., Bousier C., Abbate C., Baglieri A., and Gennari M., 2008. Use of model soil colloids to evaluate adsorption of phenanthrene and its mobilization by different solutions. J. Environ. Sci. Heal. A., 43, 1-9.
Nylander T., Samoshina Y., and Lindman B., 2006. Formation of polyelectrolyte-surfactant complexes on surfaces. Adv. Colloid Interface Sci., 123, 105-123.

Patzkó Á., 1996. Laboratory Practices in Colloid Chemistry (in Hungarian). JATEPress, Szeged, Hungary.

Ryżak M. and Bieganowski A., 2010. Determination of particle size distribution of soil using laser diffraction - comparison with areometric method. Int. Agrophys., 24, 177-181.

Ryżak M. and Bieganowski A., 2011. Methodological aspects of determining soil particle-size distribution using the laserdiffraction method. J. Plant Nutr. Soil Sci., 174, 624-633.

Sebestyén T., 2008. Study of adsorption of surfactants (in Hungarian). MSc. Thesis, University of Pannonia, Veszprém, Hungary.

Senesi N., 1993. Nature of interactions between organic chemicals and dissolved humic substances and the influence of environmental factors. In: Organic Substances in Soil and Water: Natural Constituents and Their Influences on Contaminant Behaviour (Eds A.J. Beck, K.C. Jones, M.H.B. Hayes, U. Mingelgrin). The Royal Society of Chemistry, Cambridge, UK.

Tombácz E., Szekeres M., Klumpp E., and Narres H.D., 2001. Acidic functionality of humic acid fractions prepared by a sequential ultrafiltration technique. In: Understanding and Managing Organic Matter in Soils, Sediments, and Waters (Eds R.S. Swift, K.M. Spark). IHSS, St. Paul, MI, USA.

Tombácz E., Varga K., and Szántó, F., 1988. An X-ray diffraction study of alkylammonium humate complexes. Colloid. Polym. Sci., 266, 734-738.

Tschapek M. and Wasowski C., 1984. Humic acid: its adsorption at the water/benzene interface. Agrochim., 28, 1-8.

Wandruszka R., 2000. Humic acids: Their detergent qualities and potential uses in pollution remediation. Geochem. Trans., 1, 10, doi:10.1186/1467-4866-1-10

Wilson M.S., 1991. Advances in Soil Organic Matter Research: The Impact on Agriculture and the Environment. Redwood Press Ltd., Wiltshire, UK. 